\title{
DEGENERATE AND NON-DEGENERATE GROUND STATES FOR SCHRÖDINGER OPERATORS
}

\author{
WILLIAM FARIS AND BARRY SIMON*
}

\section{Introduction.}

In the study of quantum mechanical energy operators, particular attention is paid to the question of whether the bottom of the spectrum is an eigenvalue and whether that eigenvalue is simple. The corresponding eigenvector is usually called the gound state. Throughout our discussion we will say that a self-adjoint operator $H$ has no ground state degeneracy when either $E=$ inf spectrum $H$ is not an eigenvalue or $E$ is a simple eigenvalue. Our concern in this note will be Schrödinger operators $H=-\Delta+V$ acting in $L^{2}\left(\mathbf{R}^{n}\right)$, where $V$ is a suitable multiplication operator.

When $n=1$ the lack of ground state degeneracy for a large class of $V$ is a classical theorem in the theory of ordinary differential equations. There is an argument in Courant and Hilbert [4] concerning the case of general $n$. Implicit in their arguments are assumptions (about the regularity of nodes of any eigenvector) that seem difficult to prove for general $V$. (However, we should remark that a proof along the lines of Courant and Hilbert should be possible, especially if one uses Kato's inequality [14].) The modern treatment of the non-degeneracy problem follows an idea of Glimm and Jaffe [11] in their study of quantum field models. They suggested applying theorems of PerronFrobenius $[10,17]$ type to $\exp (-t H)$. The applicability of these ideas to Schrödinger operators was noted by Simon and Höegh-Krohn [23] and Faris (see [2]). It was discussed further in Faris [8], where the following general result appears.

Theorem 1. Let $V=V_{1}+V_{2}$ where $V_{1}$ and $V_{2}$ are real functions on $\mathbf{R}^{n}$. Assume that $V_{1} \geq 0, V_{1} \in L_{100}{ }^{1}$, and that $V_{2} \in L^{\infty}+L^{p}$ where $p=n / 2$ if $n \geq 3$, $p>1$ if $n=2$, and $p=1$ if $n=1$. Then $H=-\Delta+V$ has no ground state degeneracy.

Remarks. 1. Here and below we define $H=-\Delta+V$ as a sum of quadratic forms. That is, $H$ is the unique self-adjoint operator whose quadratic form domain $\mathcal{Q}(H) \equiv \mathscr{D}\left(|H|^{\frac{1}{2}}\right)$ is $\mathcal{Q}(-\Delta) \cap \mathbb{Q}(V)$ with

$$
\left\langle(\operatorname{sgn} H)|H|^{\frac{1}{2}} \psi,|H|^{\frac{1}{2}} \psi\right\rangle=\langle\nabla \psi, \nabla \psi\rangle+\left\langle(\operatorname{sgn} V)|V|^{\frac{1}{2}} \psi,|V|^{\frac{1}{2}} \psi\right\rangle
$$

2. The conditions on $V_{2}$ are made so that $V_{2}$ is a small form perturbation

* A. Sloan fellow; research partially supported by U. S. NSF under Grant GP 39048, Received March 21, 1975. 
of $-\Delta$. The assumption on $V_{1}$ implies that $Q(-\Delta) \cap Q(V) \supset C_{0}{ }^{\infty}\left(\mathbf{R}^{n}\right)$ and so it is dense.

3. Since the $V_{2}$ part of $V$ always requires wordier statements and proofs without any significantly new ideas, our theorems below will suppose that $V_{2}=0$. It is easy to add a non-zero $V_{2}$ obeying the conditions of Therorem 1 .

Our interest in discussing improvements in Theorem 1 was raised by a general phenomenon discussed by Klauder [15] (see also [21]). Among other things, Klauder notes that $H=-\left(d^{2} / d x^{2}\right)+x^{2}+x^{-\alpha}(\alpha \geq 1)$ has a doubly degenerate ground state. The non- $L^{1}$ singularity at $x=0$ does not prevent the definition of $H$, since $C_{0}{ }^{\infty}\left(\mathbf{R}^{n} \backslash\{0\}\right) \subset Q\left(-\left(d^{2} / d x^{2}\right)\right) \cap Q\left(x^{2}+x^{-\alpha}\right)$ is dense. But it is so severe that it decouples $(-\infty, 0)$ and $(0, \infty)$. That is, $L^{2}(-\infty, 0)$ and $L^{2}(0, \infty)$ are invariant subspaces for $H$, and $H$ has identical spectrum on the two spaces and thus doubly degenerate eigenvalues.

In this note we answer the question of how general Klauder's phenomenon is. Let $K$ be a closed set of measure zero and let $V$ be positive and in $L_{1 \mathrm{oc}}{ }^{1}\left(\mathbf{R}^{n} \backslash K\right)$, so that $V$ can be very singular on $K$. We want to know under what conditions on $K$ are we assured that $H=-\Delta+V$ has no ground state degeneracy for all allowed $V$ 's. We will give necessary and sufficient conditions on $K$ by proving the following two theorems.

Theorem 2. If $\mathbf{R}^{n} \backslash K$ is connected, then $H=-\Delta+V$ cannot have a degenerate ground state for any positive $V$ in $L_{100}{ }^{1}\left(\mathbf{R}^{n} \backslash K\right)$.

THeOREm 3. If $\mathbf{R}^{n} \backslash K$ has $m$ or more components, then there is a positive $V$ in $L_{1 \mathrm{oc}}{ }^{1}\left(\mathbf{R}^{n} \backslash K\right)$ such that the ground state of $H=-\Delta+V$ is $m$-fold degenerate.

Remarks. 1. Since $K$ is a closed set of measure zero, $C_{0}{ }^{\infty}\left(\mathbf{R}^{n} \backslash K\right) \subset Q(-\Delta)$ $\cap \mathbb{Q}(V)$ is dense, and so $H=-\Delta+V$ is definable as a sum of forms.

2 . One can also prove by the methods we use that if $\mathbf{R}^{n} \backslash K$ has precisely $m$ components, then the ground state never has degeneracy larger than $m$.

We give two proofs of Theorem 2. The first (\$2) exploits path integrals and the second (\$3) decomposibility ideas which in the context of quantum mechanical problems go back to Segal [19]. One proof uses arcwise connectedness, the other topological connectedness.

\section{Path Integral Proof.}

In this section we use the machinery of path integrals and the Feynman-Kac formula. For a brief introduction, see Nelson [16] or Reed and Simon [18]; for more detailed discussion, see Ciesielski [3], Ito and McKean [12], or Kac [13]. We note that Klauder [15] has emphasized the path integral picture associated with his phenomenon.

Let $\mu_{x}$ be Wiener measure on paths $\omega$ in $\mathbf{R}^{n}$ with $\omega(0)=x$. Thus,

$$
\int f(\omega(t)) d \mu_{x}(\omega)=(\exp (t \Delta) f)(x)
$$


Let $V \geq 0$ be in $L_{\text {loe }}{ }^{1}(\Omega)$, where $\Omega \subset \mathbf{R}^{n}$ is the complement of a closed set of measure zero. Let $H=-\Delta+V$. The Feynman-Kac formula states that for $t \geq 0$ (and for suitable functions $f$ )

$$
(\exp (-t H) f)(x)=\int \exp \left(-\int_{0}^{t} V(\omega(s)) d s\right) f(\omega(t)) d \mu_{x}(\omega) .
$$

Remark. If $s>0$ and $N \subset \mathbf{R}^{n}$ is a set of measure zero, then $\mu_{x}(\omega(s) \in N)=0$. Thus $0=\int_{0}^{\infty} \mu_{x}(\omega(a) \in N) d s=\int \operatorname{meas}\{s: \omega(s) \in N\} d \mu_{x}(\omega)$. It follows that for almost every $\omega, \omega(s) \notin N$ for almost every $s$.

This implies that $\int_{0}^{t} V(\omega(s)) d s$ is unchanged if we change $V$ on a null set. It is possible that $\int_{0}^{t} V(\omega(s)) d s$ is infinite for certain paths $\omega$, but in that case we interpret $\exp \left(-\int_{0}{ }^{t} V(\omega(s)) d s\right)$ as zero for those paths.

It is easy to check the formula for the case when $V$ is in $L^{\infty}$ by expanding in a power series in $V$. The general case follows by monotone convergence for forms (see [9], for instance) and for integrals.

We need some information about paths lying in a subset of $\mathbf{R}^{n}$. Let $\Omega$ be an open set in $\mathbf{R}^{n}$ and let $\mu_{x}^{\mathbf{\Omega}}(M)=\mu_{x}(\omega \in M: \omega$ lies in $\Omega)$. Then $\Delta_{\Omega}$, the Laplacian in $\Omega$ with Dirichlet boundary conditions, may be defined by

$$
\left(\exp \left(t \Delta_{\Omega}\right) f\right)(x)=\int f(\omega(t)) d \mu_{x}^{\Omega} .
$$

Lemma 1. Let $\Omega$ be an open connected set in $\mathbf{R}^{n}$. Then exp $\left(t \Delta_{\Omega}\right)$ is an integral operator with strictly positive kernel on $L^{2}(\Omega)$.

Remark. The Dirichlet boundary conditions defined by the restriction of paths to lie in $\Omega$ agree with the conditions given by a Friedrichs extension method. While this is true for arbitrary $\Omega$ (see [3]), here we define $\Delta_{\Omega}$ in terms of the path description, and the equivalence will only be required for cubes where it is easy to establish.

Proof. It is clear from the definition that if $f \geq 0$, then $\exp \left(t \Delta_{\Omega}\right) f \geq 0$, so $\exp \left(t \Delta_{\Omega}\right)$ has a positive kernel. To show that it is strictly positive, it is sufficient to show that if meas $S>0$ and $\chi_{S}=1$ on $S, 0$ elsewhere, then

$$
\left(\exp \left(t \Delta_{\Omega}\right) \chi_{S}\right)(x)=\mu_{x}^{\Omega}(\omega(t) \in S)>0 .
$$

Consider first the case when $\Omega$ is a cube. Then it can be shown that $\Delta_{\Omega}$ is the classical Dirichlet Laplacian and by separation of variables this Laplacian has a non-degenerate ground state with strictly positive eigenfunction. By an argument of Faris [7], exp $\left(t \Delta_{\Omega}\right)$ is ergodic and then by an argument of Simon [22], it has a strictly positive kernel.

Now let $\Omega$ be arbitrary. Let $\gamma$ be a path lying in $\Omega$ such that $\gamma(0)=x$ and $\gamma(t) \in S$ essentially (that is, meas $(S \cap U)>0$ for all neighborhoods $U$ of $\gamma(t))$. Cover $\gamma$ by cubes $C_{1}, \cdots, C_{n} \subset \Omega$ such that $\gamma(0)=x \in C_{1}, \gamma(t) \in C_{n}$, and $C_{i} \cap C_{i+1}$ has non-empty interior (see Fig. 1). Since the cubes all lie in $\Omega$, we have 


$$
\mu_{x}^{\Omega}(\omega(t) \in S) \geq \mu_{x}\left(\omega(s) \in C_{i} \text { if } \frac{(j-1) t}{n} \leq s \leq \frac{j t}{n}, \text { all } j, \omega(t) \in S\right)
$$

This is the same as saying that

$$
\left(\exp \left(t \Delta_{\mathbf{\Omega}}\right) \chi_{S}\right)(x) \geq\left[\prod_{i=1}^{n}\left(\exp \left(-\frac{t}{n} \Delta_{C_{i}}\right) P_{i}\right) \chi_{s}\right](x)
$$

where $P_{j}$ is multiplication by $\chi_{C_{i}}$. But the last expression is strictly positive, by the result for cubes and the fact that the cubes overlap.

Lemma 2. Let $\Omega$ be an open set in $\mathbf{R}^{n}$. Let $V \geq 0$ be locally $L^{1}$ in $\Omega$. Fix $t>0$. Then for almost every $x$ and almost all paths $\omega$ starting at $x$ and lying in $\Omega, \int_{0}{ }^{t} V(\omega(s)) d s<\infty$.

Proof. Let $\Omega_{m}$ be a sequence of compact sets with $\Omega_{m} \subset \Omega_{m+1}{ }^{\text {int }}$ and $\cup{ }_{m} \Omega_{m}=\Omega$. Let $V_{m}=V \chi_{m}$, where $\chi_{m}=1$ on $\Omega_{m}, 0$ elsewhere. Thus $V_{m} \in L^{1}$ for each $m$.

Since the unrestricted Wiener measure is translation invariant, we have

$$
\int V_{m}(\omega(s)) d \mu_{x}(\omega)=\int V_{m}(x+\omega(s)) d \mu_{0}(\omega) .
$$

Hence

$$
\int d x \int V_{m}(\omega(s)) d \mu_{x}(\omega)=\int d \mu_{0}(\omega) \int V_{m}(x+\omega(s)) d x=\int V_{m}(x) d z<\infty .
$$

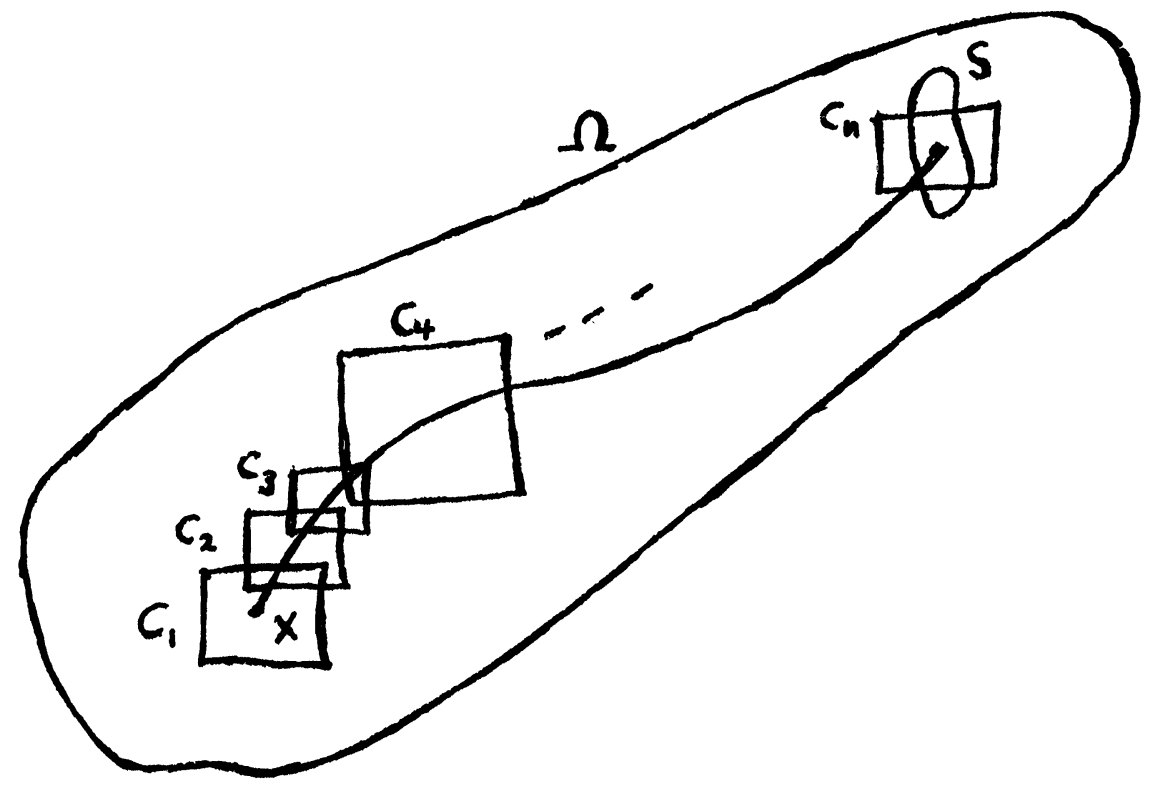

Figure 1 
It follows from Fubini's theorem that

$$
\int d x \int d \mu_{x}(\omega) \int_{0}^{t} V_{m}(\omega(s)) d s=t \int V_{m}(x) d x<\infty
$$

and hence for almost every $x \in \mathbf{R}^{n}$ and almost every path in $\mathbf{R}^{n}$,

$$
\int_{0}^{t} V_{m}(\omega(s)) d s<\infty .
$$

In particular, for almost every $x \in \mathbf{R}^{n}$ and almost every path lying in $\Omega_{m}$, $\int_{0}{ }^{t} V(\omega(s)) d s<\infty$. Since the paths are continuous, almost every path lying in $\Omega$ lies in some $\Omega_{m}$. Hence for almost every $x$ and almost every path lying in $\Omega, \int_{0}{ }^{t} V(\omega(s)) d s<\infty$.

Proof of Theorem 2. By the Feynman-Kac formula, if $f \geq 0$ then $\exp (-t H) f$ $\geq 0$. Thus $\exp (-t H)$ has a positive kernel. We will show that it has a strictly positive kernel by showing that if meas $S>0$, then $\exp (-t H) \chi_{S}$ is strictly positive almost everywhere.

Let $\Omega=\mathbf{R}^{n} \backslash K$. Since the set of all paths from $x$ to $S$ which lie in $\Omega$ is a subset of the set of all paths from $x$ to $S$,

$$
\begin{aligned}
\left(\exp (-t H) \chi_{S}\right)(x)=\int_{\omega(t) \in S} \exp \left(-\int_{0}^{t} V(\omega(s)) d s\right) d \mu_{x}(\omega) & \\
& \geq \int_{\omega(t) \in S} \exp \left(-\int_{0}^{t} V(\omega(s)) d s\right) d \mu_{x}{ }^{\Omega}(\omega) .
\end{aligned}
$$

Since $\Omega$ is open and connected, by lemma $1 \mu_{x}^{\Omega}(\omega(t) \in S)>0$. But since $V$ is in $L^{1}$ locally in $\Omega$, by lemma 2 , for almost all $x$ and almost all $\omega$ starting at $x$ and lying in $\Omega, \int_{0}{ }^{t} V(\omega(s)) d s<\infty$. Thus for almost all $x$ the integrand of the path integral is strictly positive on a set of strictly positive measure, and hence the integral is strictly positive.

The proof is concluded by noting that semigroups $\exp (-t H)$ with strictly positive kernels have generators $H$ without ground state degeneracy [11].

Proof of Theorem 3. Let $V_{0}(x)=x^{2}+[\operatorname{dist}(x, K)]^{-3}$. Then $V_{0} \in L_{100}{ }^{1}\left(\mathbf{R}^{n} \backslash K\right)$ Moreover, since $-\Delta+V_{0} \geq-\Delta+x^{2},-\Delta+V_{0}$ has purely discrete spectrum. Let $x$ and $y$ be in distinct components of $\mathbf{R}^{n} \backslash K$. Let $\omega$ be a path from $x$ to $y$ which is Hölder continuous of order $\frac{1}{3}$. Then since $\omega$ must go through $K$ to get from $x$ to $y, \int \operatorname{dist}(\omega(t), K)^{-3} d t=\infty$. So for $x$ and $S$ in distinct components of $\mathbf{R}^{n} \backslash K$, the Feynman-Kac formula gives

$$
\left(\exp \left(-t\left(-\Delta+V_{0}\right)\right) \chi_{S}\right)(x)=\int_{\omega(t) \in S} \exp \left(-\int_{0}^{t} V_{0}(\omega(s)) d s\right) d \mu_{x}(\omega)=0 .
$$

Thus if $\Omega_{1}, \cdots, \Omega_{m-1}$ are distinct components of $\mathbf{R}^{n} \backslash K$ and $\Omega_{m}$ is the union of the other components, then $L^{2}\left(\mathbf{R}^{n} \backslash K\right)=\bigoplus_{k=1}^{m} L^{2}\left(\Omega_{k}\right)$ and under this decomposition $\exp \left(-t\left(-\Delta+V_{0}\right)\right)=e^{-t A_{1}} \oplus \cdots \oplus e^{-t A_{m}}$. Now $A_{1}, \cdots, A_{m}$ all have discrete spectrum. So by adding suitable constants $c_{1}, \cdots, c_{m}$ to 
$A_{1}, \cdots, A_{m}$ we can ensure that $\left(A_{1}+c_{1}\right) \oplus \cdots \oplus\left(A_{m}+c_{m}\right)$ has an at least $m$-fold degenerate ground state. We let $V=V_{0}+\sum_{k=1}^{m} c_{k} \chi_{k}$, where $\chi_{k}$ is 1 on $\Omega_{k}, 0$ elsewhere. Then $H=-\Delta+V$ has an at least $m$-fold degenerate ground state.

Remark. Since Wiener paths are Hölder continuous of order $\frac{1}{2}-\epsilon, \operatorname{dist}(x, K)^{-3}$ can be replaced by $\operatorname{dist}(x, K)^{-2-\epsilon}$ without any change in the proof. Actually we expect that, at least for reasonable $K$, $\operatorname{dist}(x, K)^{-1}$ will suffice. Our expectation is based on two facts. (a) Quadratic form considerations suggest that if $\psi \in Q(-\Delta)$ and $\int \operatorname{dist}(x, K)^{-1}|\psi(x)|^{2}<\infty$, then $\psi=0$ on $K$, at least if $K$ is a smooth manifold of codimension 1 (see Agmon [1]). Thus the quadratic form mechanism that causes Klauder's phenomenon in one dimension to occur at $x^{-1}$ potentials [21] should work in more than one dimension. (b) In one dimension it has been proven that almost every path from $x=1$ to $x=-1$ has $\int|\omega(t)|^{-1} d t=\infty[5,6]$.

\section{Differential Operator Proof.}

Our second proof of Theorem 2 rests on the following criterion for nondegeneracy of ground states (see Segal [20], Simon and Höegh-Krohn [23] or Faris [9]).

Definition. A configuration projection is a projection in $L^{2}(M, \nu)$ which is a multiplication operator. A bounded operator is called indecomposable if it commutes with no configuration projections other than the trivial ones 0 and 1 .

Lemma 3. If $H$ is a semi-bounded, self-adjoint operator acting in $L^{2}(M, \nu)$ such that $\exp (-t H)$ is positivity preserving and indecomposable, then $H$ has no ground state degeneracy.

We also need a basic perturbation lemma.

LEMma 4. Let $H_{0}$ and $U$ be positive self-adjoint operators acting in $L^{2}(M, \nu)$. Suppose $\mathbb{Q}(H)=\mathbb{Q}_{(}\left(H_{0}\right) \cap \mathbb{Q}(U)$ is dense. Let $H=H_{0}+U$ as a sum of forms on $Q(H)$ and let $\hat{H}_{0}$ be the self-adjoint operator whose form is the closure of the restriction of $H_{0}$ to $Q(H)$. Suppose that $U$ is a multiplication operator. Then $\exp (-H)$ is indecomposable if and only if $\exp \left(-\hat{H}_{0}\right)$ is indecomposable.

Proof. Let $U_{n}=\min (U, n)$. Then it is easy to see using monotone convergence theorems for forms [9] that $\hat{H}_{0}+U_{n} \rightarrow H$ in the strong resolvent sense and that $H-U_{n} \rightarrow \hat{H}_{0}$ in the strong resolvent sense. Thus by the Trotter product formula

$$
\begin{aligned}
& \exp (-t H)=\lim _{n \rightarrow \infty} \lim _{m \rightarrow \infty}\left(\exp \left(-t \hat{H}_{0} / m\right) \exp \left(-t U_{n} / m\right)\right)^{m} \\
& \exp \left(-t \hat{H}_{0}\right)=\lim _{n \rightarrow \infty} \lim _{m \rightarrow \infty}\left(\exp (-t H / m) \exp \left(+t U_{n} / m\right)\right)^{m}
\end{aligned}
$$

with strong convergence. It follows that a configuration projection commutes with $\exp (-t H)$ if and only if it commutes with $\exp \left(-t \hat{H}_{0}\right)$. But by the func- 
tional calculus, for any semi-bounded, self-adjoint operator $A$, a projection $P$ commutes with $\exp (-t A)$ for all $t$ if and only if it commutes with $\exp (-A)$.

Proof of Theorem 2. Consider $V \geq 0$ in $L_{\text {loc }}{ }^{1}\left(\mathbf{R}^{n} \backslash K\right)$. Let $V_{n}=\min (V, n)$. Then $H=-\Delta+V=\lim _{n \rightarrow \infty}\left(-\Delta+V_{n}\right)$ (strong resolvent limit), by a monotone convergence theorem for forms. Hence

$$
\exp (-t H)=\lim _{n \rightarrow \infty} \lim _{m \rightarrow \infty}\left(\exp (t \Delta / m) \exp \left(-t V_{n} / m\right)\right)^{m}
$$

(strong limit), so $\exp (-t H)$ is positivity preserving. Thus by Lemma 3 it is sufficient to prove that $\exp (-H)$ is indecomposable. But by Lemma 4 for this we need only prove that $\exp \left(-\hat{H}_{0}\right)$ is indecomposable.

Now $\hat{H}_{0}$ is a quadratic form whose form domain contains $C_{0}{ }^{\infty}\left(\mathbf{R}^{n} \backslash K\right)$ and is contained in $Q(-\Delta)$. It follows that $C_{0}{ }^{\infty}\left(\mathbf{R}^{n} \backslash K\right) \subset \mathbb{D}\left(\hat{H}_{0}\right)$ and the operators $\hat{H}_{0}$ and $-\Delta$ have the same restrictions to $C_{0}{ }^{\infty}\left(\mathbf{R}^{n} \backslash K\right)$.

Let $P$ be a configuration projection commuting with $\exp \left(-\hat{H}_{0}\right)$. Then $P$ is multiplication by some function $\chi_{S}$. Since $D\left(\hat{H}_{0}\right)=\left\{\phi \mid \exp \left(-t \hat{H}_{0}\right) \phi\right.$ is $C^{\mathbf{1}}$ at $t=0\}, \phi \in \mathbb{D}\left(\hat{H}_{0}\right)$ implies that $\chi_{S} \phi \in \mathbb{D}\left(\hat{H}_{0}\right)$ and $\hat{H}_{0}\left(\chi_{s} \phi\right)=\chi_{S}\left(\hat{H}_{0} \phi\right)$.

Define $\operatorname{supp} S=\{x \mid$ meas $(S \cap N) \neq 0$ for every neighborhood $N$ of $x\}$. Let $\partial S=\operatorname{supp} S \cap \operatorname{supp}\left(\mathbf{R}^{n} \backslash S\right)$. We claim that $\partial S \subset K$. Let $x \notin K$. Choose $\phi \in C_{0}{ }^{\infty}\left(\mathbf{R}^{n} \backslash K\right)$ so that $\phi=1$ near $x$. Since $\phi \in \mathbb{D}\left(\hat{H}_{0}\right), \chi_{S} \phi \in \mathbb{D}\left(\hat{H}_{0}\right)$, so $\hat{H}_{0}\left(\chi_{S} \phi\right)=\chi_{S}\left(\hat{H}_{0} \phi\right)=\chi_{S}(-\Delta \phi)=0$ near $x$. Thus $\Delta \chi_{S}=0$ near $x$. By the elliptic regularity theorem, $\chi_{S}$ is, after change on a set of measure $0, C^{\infty}$ near $x$, so $x \notin \partial S$.

Thus supp $S \cap \operatorname{supp}\left(\mathbf{R}^{n} \backslash S\right) \subset K$. Since supp $S \cup \operatorname{supp}\left(\mathbf{R}^{n} \backslash S\right)=\mathbf{R}^{n}$ and the support is closed, the hypothesis that $\mathrm{R}^{n} \backslash K$ is connected implies that either supp $S \subset K$ or supp $\left(\mathbf{R}^{n} \backslash S\right) \subset K$. Since $K$ has measure zero, $P=0$ or $P=1$. Thus $\exp \left(-t \hat{H}_{0}\right)$ is indecomposable.

Remark. There is an alternative proof of Lemma 4 which uses the operators directly instead of going through the semigroups [9]. A variant of this idea may be used to show that $H=-\Delta+G$ cannot have ground state degeneracy even in certain cases when $G$ is not a multiplication operator.

Theorem 4. Assume that $-G$ is positivity preserving and that $G$ is relatively bounded with respect to $\Delta$. Then $H=-\Delta+G$ cannot have ground state degeneracy.

Proof. A perturbation argument [7] shows that $\exp (-H)$ is positivity preserving. In order to be able to apply Lemma 3 we need to show that it is also indecomposable.

Let multiplication by $\chi$ be a configuration projection which commutes with $\exp (-H)$. It must also commute with $H$ and in particular leave $\mathfrak{D}(H)$ invariant. But since $G$ is relatively bounded, $D(H)=D(\Delta)$, so it also leaves $D(\Delta)$ invariant.

If $x \in \mathbf{R}^{n}$ and $\phi$ is smooth with $\phi=1$ near $x$, then $\chi \phi$ is in $D(\Delta)$ and $\chi \phi=\chi$ near $x$. Hence $\Delta \chi \in L^{2}$ near $x$. By Lemma $5, \chi$ is constant. This proves indecomposability. 
Lemma 5. If $\chi$ on $\mathbf{R}^{n}$ is 2-valued and $\Delta \chi \in L_{1 \mathrm{oc}}{ }^{2}\left(\mathbf{R}^{n}\right)$, then $\chi$ is equal almost everywhere to a constant function.

Proof. We suppose that $\chi$ takes on the values 0 and 1 . Since $D(-\Delta) \subset$ $D\left(\partial^{2} / \partial x_{1}{ }^{2}\right) \cap \cdots \cap D\left(\partial^{2} / \partial x_{n}{ }^{2}\right)$, we know that $\partial^{2} \chi / \partial x_{k}{ }^{2} \in L_{1 \mathrm{oc}}{ }^{2}\left(\mathbf{R}^{n}\right)$. In particular $\partial \chi / \partial x_{k} \in L_{10 \mathrm{oc}}{ }^{2}\left(\mathbf{R}^{n}\right) \subset L_{\mathrm{loc}}{ }^{1}\left(\mathbf{R}^{n}\right)$.

This implies that for almost every $y_{k}=\left(x_{1}, \cdots, x_{k-1}, x_{k+1}, \cdots, x_{n}\right)$, $\partial \chi / \partial x_{k} \in L_{1 \mathrm{oc}}{ }^{1}\left(\mathbf{R}^{n}\right)$ as a function of $x_{k}$. Hence for almost every $y_{k} \in \mathbf{R}^{n-1}$, $\chi$ is equal almost everywhere to a continuous function of $x_{k}$. For each $y_{k}$ this function must be 0 or 1 . Let $\chi_{k}$ be the function on $\mathbf{R}^{n}$ which for fixed $y_{k}$ is equal to this corresponding constant function of $x_{k}$. Then for almost every $y_{k} \in \mathbf{R}^{n-1}, \chi$ is equal to $\chi_{k}$ for almost every $x_{k}$. Thus $\chi$ is equal to $\chi_{k}$ almost everywhere.

Since this is true for each $k$, it follows that $\chi=\chi_{1}=\cdots=\chi_{n}$ almost everywhere. Since each $\chi_{k}$ is independent of $x_{k}, \chi=$ const almost everywhere.

\section{REFERENCES}

1. S. Agmon, Spectral properties of Schrödinger operators and scattering theory, Scuola Normale Supariore Pisa (lecture notes).

2. N. BazLey and B. ZWahlen, A branch of positive solutions of non-linear eigenvalue problems, Manuscripta Math. 2 (1970), 365-374.

3. Z. Ciesielski, Lectures on Brownian Motion, Heat Conduction, and Potential Theory, Aarhus Lecture Notes, Series No. 3.

4. R. Courant and D. Hilbert, Methods of Mathematical Physics, Vol. I, Interscience, 1953.

5. H. Ezawa, J. R. KLauder, and L. A. Shepp, Distributions aleatoires et les processus Gaussions, Proc. Strasbourg Colloquium, July, 1973.

6. - Vestigial effects of singular potentials in diffusion theory and quantum mechanics, Bell Labs. (preprint).

7. W. FARIs, Invariant cones and uniqueness of the ground state for fermion systems, J. Mathematical Phys. 13 (1972), 1285-1290.

8. - Q Quadratic forms and essential self-adjointness, Helv. Phys. Acta 45 (1972), 1074-1088.

9. ——, Self-adjoint Operators (Lecture Notes in Mathematics \#433), Springer-Verlag, Heidelberg, 1975.

10. G. Frobenius, Uber Matrixen mit positiven Elementen, Sitz. d. K. Preuss. Akad. d. Wissen., Berlin (1908), 471-476.

11. J. Gumm And A. JAFFe, The $\lambda\left(\phi^{4}\right)_{2}$ quantum field theory without cutoffs II, Ann. Math. 91 (1970), 362-401.

12. K. Ito and H. P. McKean, Diffusion Processes and Their Sample Paths, Springer, New York, 1965.

13. M. KAc, Probability and Related Topics in the Physical Sciences, Interscience, New York, 1959.

14. T. KAтo, Schrödinger operators with singular potentials, Israel J. Math. 13 (1972), 135148.

15. J. Klauder, Field structure through model studies, Acta Phys. Aust. Suppl. XI (1973), 341.

16. E. Nelson, Feynman integrals and the Schrödinger equation, J. Mathematical Phys. 5 (1964), 332-343.

17. O. Perron, Zur Theorie der Matrizen, Math. Ann. 64 (1907), 248-263. 
18. M. Reed and B. Simon, Methods of Modern Mathematical Physics, Vol. II Fourier Transforms, Self-adjointness, Academic Press, 1975.

19. I. Segal, Notes towards the construction of non-linear relativistic quantum fields III, Bull. Amer. Math. Soc. 75 (1969), 1390-1395.

20. - Construction of non-linear local quantum processes: II, Invent. Math. 14 (1971), 211-241.

21. B. Srmon, Quadratic forms and Klauder's phenomenon: A remark on very singular perturbations, J. Functional Anal. 14 (1973), 295-298.

22. ——_ Ergodic semigroups of positivity preserving self-adjoint operators, J. Functional Anal. 12 (1973), 335-339.

23. B. Simon and R. HöEgh-Krohn, Hypercontractive semigroups and two-dimensional selfcoupled Bose fields, J. Functional Anal. 9 (1972), 121-180.

Faris: Department of Mathematics, University of Arizona, Tucson, Arizona 85721

Simon: Departments of Mathematics and Physics, Princeton University, Princeton, New Jersey 08540 